Pacific Journal of Mathematics

A CONSTRUCTION OF INNER MAPS PRESERVING THE 


\title{
A CONSTRUCTION OF INNER MAPS PRESERVING THE HAAR MEASURE ON SPHERES
}

\author{
BOGUSLAW TOMASZEWSKI
}

\begin{abstract}
We show, for $n \geq m$, the existence of non-trivial inner maps $f$ : $B^{n} \rightarrow B^{m}$ with boundary values $f_{*}: S^{n} \rightarrow S^{m}$ such that $f_{*}^{-1}(A)$ has a positive Haar measure for every Borel subset $A$ of $S^{m}$ which has a positive Haar measure. Moreover, if $n=m$, the equality $\sigma\left(f_{*}^{-1}(A)\right)=$ $\sigma(A)$ holds, where $\sigma$ is the Haar measure of $S^{m}$.
\end{abstract}

In this paper $\mathbf{C}^{n}$ is an $n$-dimensional complex space with inner product defined by $\left\langle z^{1}, z^{2}\right\rangle=\sum z_{i}^{1} \bar{z}_{i}^{2}$, where $z^{j}=\left(z_{1}^{j}, z_{2}^{j}, \ldots, z_{n}^{j}\right)$ for $j=$ 1,2 , and the norm $|z|=\langle z, z\rangle^{1 / 2}$. Let us introduce some notation:

$$
B^{n}=\left\{z \in \mathbf{C}^{n}:|z|<1\right\}, \quad S^{n}=\partial B^{n} ;
$$

let $d$ be the metric on $S^{n}$ :

$$
d\left(z, z^{*}\right)=\left(1-\operatorname{Re}\left\langle z, z^{*}\right\rangle\right)^{1 / 2}=\frac{1}{\sqrt{2}}\left|z-z^{*}\right| \quad \text { for } z, z^{*} \in S^{n},
$$

and finally

$$
B(z, r)=\left\{z^{*} \in S^{n}: d\left(z, z^{*}\right)<r\right\} \quad \text { for } z \in S^{n} \text { and } r>0 .
$$

For every complex function $h: X \rightarrow \mathbf{C}$ we define $Z(h)=h^{-1}(0)$. A holomorphic map $f: B^{n} \rightarrow B^{m}$ is called inner if

$$
f_{*}(z)=\lim _{r \rightarrow 1} f(r z) \in S^{m} \quad \text { for almost every } z \in S^{n}
$$

with respect to the unique, rotation-invariant Borel measure $\sigma_{n}$ on $S^{n}$ such that $\sigma_{n}\left(S^{n}\right)=1$. If a continuous function $g: \bar{B}^{n} \rightarrow \mathbf{C}^{m}$, defined on the closure of $B^{n}$, is holomorphic on $B^{n}$, we write $g \in A_{m}\left(B^{n}\right)$ or $g \in A\left(B^{n}\right)$ when $m=1$. The theorem stated below is a generalization of the result of Aleksandrov [1]. Corollary 1 answers the problem given by Rudin [3]. Corollary 4 is a result of Aleksandrov obtained independently by the author.

THEOREM. Let $n \geq m$ and let $g=\left(g_{1}, \ldots, g_{m}\right) \in A_{m}\left(B^{n}\right), h \in A\left(B^{n}\right)$ be maps such that $|g(z)|+|h(z)| \leq 1$ and $h(z) \neq 0$ for some $z \in B^{n}$. Then there exists an inner map $f=\left(f_{1}, f_{2}, \ldots, f_{m}\right): B^{n} \rightarrow B^{m}$ such that $f(z)=$ $g(z)$ for every $z \in Z(h)$ and $f_{i}(z)=g_{i}(z)$ for every $z \in B^{n}$ and $i=$ $1,2, \ldots, m-1$. 
COROllaRY 1. For every $n \geq m$ there exist inner maps $f: B^{n} \rightarrow B^{m}$ such that for every Borel subset $A \subset S^{m}$ the inequality $\sigma_{n}\left(f_{*}^{-1}(A)\right)>0$ holds provided $\sigma_{m}(A)>0$. Moreover, if $m=n$, the equality $\sigma_{n}\left(f_{*}^{-1}(A)\right)=\sigma_{n}(A)$ holds and $f$ is not an automorphism of $B^{n}$.

COROllaRy 2. For every $n \geq 1$ there exist inner maps $f: B^{n} \rightarrow B^{m}$, not automorphisms of $B^{n}$, such that

$$
\int_{S^{n}}\left(h \circ f_{*}\right) d \sigma_{n}=\int_{S^{n}} h d \sigma_{n}
$$

for every continuous function $h$ on $S^{n}$.

Corollary 2 is an immediate consequence of Corollary 1. Let us assume that $n \geq m$ and $n \geq 2$. To deduce the assertion of Corollary 1 from the Theorem let us take a holomorphic function $k \in A\left(B^{1}\right)$ and the map $g \in A_{m}\left(B^{n}\right), g(z)=p(z)+\frac{1}{4} z_{n}^{2} r\left(z_{n}\right)$, where $p(z)=\left(z_{1}, z_{2}, \ldots\right.$, $\left.z_{m-1}, 0\right), r(z)=\left(0, \ldots, 0, k\left(z_{n}\right)\right)$ for $z \in B^{n}$. Define $h(z)=\frac{1}{4} z_{1} z_{n}^{2}$. Then

$$
|g(z)|+|h(z)| \leq|p(z)|+\frac{1}{4}\left|z_{n}^{2}\right|+\frac{1}{4}\left|z_{n}^{2}\right| \leq \sqrt{1-z_{n}^{2}}+\frac{1}{2}\left|z_{n}^{2}\right| \leq 1 .
$$

By virtue of the Theorem there exists an inner map $f=\left(f_{1}, f_{2}, \ldots, f_{m}\right)$ : $B^{n} \rightarrow B^{m}$ such that

$$
\begin{aligned}
& f_{j}\left(z_{1}, z_{2}, \ldots, z_{n}\right)=z_{j} \text { for } j=1,2, \ldots, m-1, \\
& f_{m}\left(0,0, \ldots, 0, z_{n}\right)=\frac{1}{4} z_{n}^{2} r\left(z_{n}\right), \\
& f\left(z_{1}, z_{2}, \ldots, z_{n-1}, 0\right)=\left(z_{1}, z_{2}, \ldots, z_{m-1}, 0\right) .
\end{aligned}
$$

For any $z \in B^{m-1}$ and $l \geq m$ let

$$
\begin{aligned}
& B_{z}^{l}=\left\{z^{*} \in B^{\prime}: z_{j}^{*}=z_{j} \text { for } j=1,2, \ldots, m-1\right\}, \\
& S_{z}^{l}=\left\{z^{*} \in S^{l}: z_{j}^{*}=z_{j} \text { for } j=1,2, \ldots, m-1\right\},
\end{aligned}
$$

let $\sigma_{z}^{l}$ be the rotation-invariant measure on the sphere $S_{z}^{l}$ such that $\sigma_{z}^{l}\left(S_{z}^{l}\right)=1$ and let $f_{z}, f_{z}^{*}$ be the restrictions of $f, f_{*}$ to the sets $B_{z}^{n}$ and $S_{z}^{n}$ respectively. From (1) it follows that $f_{z}: B_{z}^{n} \rightarrow B_{z}^{m}$ and (2) says that $f_{z}\left(w_{1}\right)=w_{2}$, where $w_{1}, w_{2}$ are the centers of the balls $B_{z}^{n}, B_{z}^{m}$ respectively. Since $B_{z}^{m}$ is a one-dimensional complex ball, the equality $\sigma_{z}^{n}\left(\left(f_{z}^{*}\right)^{-1}(C)\right)$ $=\sigma_{z}^{m}(C)$ holds for every Borel subset $C$ of $S_{z}^{m}$ and every $z$ for which $f_{z}$ is an inner map (see [4] p. 405). The function $f_{z}$ is inner for almost every $z \in B^{m-1}$ (with respect to the usual Lebesgue measure $\lambda$ on $B^{m-1}$ ) because the map $f$ is inner. Let us notice that there are positive functions 
$s_{1}, s_{2}: B^{m-1} \rightarrow R_{+}$such that for all Borel subsets $C^{1} \subset S^{n}, C^{2} \subset S^{m}$ we have

$$
\begin{aligned}
& \sigma_{n}\left(C^{1}\right)=\int_{B^{m-1}} s_{1}(z) \cdot \sigma_{z}^{1}\left(C_{z}^{1}\right) d \lambda(z), \\
& \sigma_{m}\left(C_{2}\right)=\int_{B^{m-1}} s_{2}(z) \cdot \sigma_{z}^{m}\left(C_{z}^{2}\right) d \lambda(z),
\end{aligned}
$$

where $C_{z}^{1}=C^{1} \cap S^{n}, C_{z}^{2}=C^{2} \cap S^{m}$. Substituting $C_{1}=\left(f^{*}\right)^{-1}\left(C_{2}\right)$ and using the equality $\sigma_{z}^{n}\left(C_{z}^{1}\right)=\sigma_{z}^{m}\left(C_{z}^{2}\right)$ (which holds for almost every $z$ ), it is easy to see that both of the above integrals are positive or equal to 0 . If $n=m$ then $s_{1}=s_{2}$ and the equality holds. This ends the proof of Corollary 1.

The following proof of the assertion of the Theorem is based on Löw's construction of inner functions [3]. Let $g$ and $h$ be maps satisfying the assumptions of the Theorem. Then $\sigma_{n}(F)=0$, where $F=Z(h) \cap S^{n}$. (This fact can be proved by induction. For $n=1$ it is well-known theorem.) For $\delta>0$ let

$$
F_{\delta}=\left\{z \in S^{n}: d(z, F)<\delta\right\} \quad \text { and } \quad\left\|s\left|\|_{\delta}=\sup _{z \in F_{\delta}}\right| s(z) \mid,\right.
$$

where $s: S^{n} \rightarrow \mathbf{C}^{m}$ is a continuous map. Observe that there exist constants $A_{1}, A_{2}$ such that for every $0<r<\sqrt{2}$,

$$
A_{1} r^{2 n-1} \leq A(r) \leq A_{2} r^{2 n-1},
$$

where $A(r)=\sigma_{n}(B(z, r))$ for any $z \in S^{n}$.

Let $S \subset S^{n}$ be any closed subset of $S^{n}, \sigma_{n}(S)>0$. Assume that for some number $r>0$,

$$
\sigma_{n}\left(S_{r}\right) \leq 2 \sigma_{n}(S)
$$

where $S_{r}=\left\{z \in S^{n}: d(z, S)<r\right\}$. Let $\left\{B\left(z^{J}, r\right)\right\}_{j=1}^{N(r)}$ be a maximal family of disjoint balls with centers $z^{J} \in S$. Since $S_{r} \supset \bigcup_{j=1}^{N(r)} B\left(z^{J}, r\right)$ and $S \subset \bigcup_{j=1}^{N(r)} B\left(z^{J}, 2 r\right)$, applying inequalities (4) and (5), we get

$$
\begin{aligned}
2 \sigma_{n}(S) & \geq \sigma_{n}\left(S_{r}\right) \geq \sigma_{n}\left(\bigcup_{j=1}^{N(r)} B\left(z^{j}, r\right)\right)=\sum_{j=1}^{N(r)} \sigma_{n}\left(B\left(z^{j}, r\right)\right) \\
& =N(r) \cdot A(r) \geq A_{1} r^{2 n-1} \cdot N(r)
\end{aligned}
$$


and

$$
\begin{aligned}
\sigma_{n}(S) & \leq \sigma_{n}\left(\bigcup_{j=1}^{N(r)} B\left(z^{j}, 2 r\right)\right)=\sum_{j=1}^{N(r)} A(2 r)=N(r) \cdot A(2 r) \\
& \leq N(r) \cdot A_{2} \cdot(2 r)^{2 n-1}=N(r) \cdot A_{2} \cdot 2^{2 n-1} \cdot r^{2 n-1} .
\end{aligned}
$$

So we have proved the existence of positive constants $C_{1}$ and $C_{2}\left(C_{1}=\right.$ $\left.1 / 2^{2 n-1}, C_{2}=2 / A_{1}\right)$ such that

$$
\frac{C_{1}}{r^{2 n-1}} \cdot \sigma_{n}(S) \leq N(r) \leq \frac{C_{2}}{r^{2 n-1}} \cdot \sigma_{n}(S) .
$$

Let us assume now that $r>0, z \in B^{n}, k$ is a natural number and $M_{k}$ is the maximal number of disjoint balls of radius $r$ and with centers in $B(z,(k+1) r)$. Because these balls are included in $B(z,(k+2) r)$, an argument similar to the above gives the estimate

$$
M_{k} \leq C_{3} k^{2 n-1}
$$

for some constant $C_{3}$. Let $\varphi:(0,1) \rightarrow R$ be the continuous, positive function defined by

$$
\varphi(a)=\frac{1}{4 \pi} \cdot C_{1} \cdot A_{1} \cdot \arccos (a) \cdot\left[\log \frac{1}{a}\right]^{(2 n-1) / 2} .
$$

Lemma 1. Let $0<2 \varepsilon<a<b, 0<\delta<2 C_{3} \cdot a, \varepsilon<C_{3} e^{-2 n}, R<1$. Let $P$ be a closed subset of $F_{\delta}$ and let $v$ be a continuous map $v: S^{n} \rightarrow \mathbf{C}^{m}$ such that $|v(z)|>$ a for $z \in P$. There exists a closed subset $K$ of $F_{\delta}$ and $a$ holomorphic map $u: \mathbf{C}^{n} \rightarrow \mathbf{C}^{m}$ such that:

$$
\begin{aligned}
& \|v+h \cdot u\|_{\delta / 2} \leq \max \left(1,\|f \mid\|_{\delta / 2}\right)+3 \varepsilon \\
& \|u\|_{R}=\sup _{|z| \leq R}|u(z)| \leq \varepsilon \\
& |v(z)+h(z) \cdot u(z)|>a-3 \varepsilon \quad \text { for } z \in K \cup P \\
& K \subset F_{\delta}, \quad K \cap P=0 \text { and } \\
& \sigma_{n}(K) \geq \varphi(a) \cdot\left[\log \left(4 C_{3} / \delta \varepsilon\right)\right]^{-(2 n-1) / 2} \cdot \sigma_{n}\left(F_{\delta}-P\right) \\
& |g(z)|<\varepsilon \quad \text { for } z \in B^{n}-F_{\delta / 2} ; \\
& u_{j} \equiv 0 \text { for } j=1,2, \ldots, m-1, \text { where } u=\left(u_{1}, u_{2}, \ldots, u_{m}\right) .
\end{aligned}
$$

Proof. If $\sigma_{n}(P)=\sigma_{n}\left(F_{\delta}\right)$ then the map $u=(0,0, \ldots, 0)$ and the set $K=\varnothing$ satisfy conditions (a)-(e). Let us assume that $\sigma_{n}(P)<\sigma_{n}\left(F_{\delta}\right)$. 
There exists a positive number $\gamma$ such that $\gamma<\delta / 2$ and

$$
\sigma_{n}(S) \geq \frac{1}{2} \cdot \sigma_{n}\left(F_{\delta}-P\right),
$$

where $S=S^{n}-\left[\left(S^{n}-F_{\delta}\right) \cup P\right]_{\gamma}$.

Since $v, h$ are uniformly continuous maps and $S$ is a closed subset, there exists a positive number $\gamma^{*}$ such that

$$
\begin{aligned}
\left|g(z)-g\left(z^{\prime}\right)\right|<\varepsilon \delta, \quad & \left|v(z)-v\left(z^{\prime}\right)\right|<\varepsilon, \quad \sigma_{n}\left(S_{r}\right) \leq 2 \cdot \sigma_{n}(S) \\
& \text { for } z, z^{\prime} \in S^{n}, d\left(z, z^{\prime}\right)<\gamma^{*} \text { and } r<\gamma^{*} .
\end{aligned}
$$

Let $r, m$ be positive numbers such that $r \leq \frac{1}{2} \min \left(\gamma, \gamma^{*}\right), m$ is an integer and $m r^{2}=\log \left(2 C_{3} / \delta \varepsilon\right)$. Moreover we assume $m$ is large so that

$$
C_{2} \cdot m^{(2 n-1) / 2} \cdot e^{-m(1-R)}<\varepsilon .
$$

Choose a maximal family $\left\{B\left(z^{j}, r\right)\right\}_{j=1}^{N(r)}$ of pairwise disjoint balls with centers $z^{j} \in S^{n}$. Because of (9), condition (5) is satisfied, so inequalities (6) also hold. For $k=1,2, \ldots,[\sqrt{2} / r]$ and $z \in S^{n}$ let

$$
V_{k}(z)=\left\{z^{\jmath}: k r \leq d\left(z, z^{\jmath}\right)<(k+1) r\right\}
$$

and let $N_{k}(z)$ be the number of elements of the set $V_{k}$. Since $V_{k}(z) \subset$ $B(z,(k+1) r)$, from the definition of $M_{k}$, we have $N_{k}(z) \leq M_{k}$ and (7) gives us

$$
N_{k}(z) \leq C_{3} k^{2 n-1} .
$$

Let $g(z)=\sum_{J=1}^{N(r)} \beta_{J} e^{-m\left(1-\left(\left\langle z, z^{J}\right\rangle\right)\right)}$, where $\beta_{j}=\left(0,0, \ldots, 0, \alpha_{J}\right) \in \mathbf{C}^{m}$ is defined by $\beta_{J}=(0,0, \ldots, 0,0)$ if $\left|f\left(z^{J}\right)\right| \geq b$. If $\left|f\left(z^{J}\right)\right|<b$, then let $\beta$, be of the previous form, such that

$$
\left|f\left(z^{j}\right)+h(z) \cdot \beta_{j}\right|=b \text { and }\left|f\left(z^{j}\right)+\alpha \cdot h(z) \cdot \beta_{j}\right| \leq b
$$

for every $\alpha \in \mathbf{C},|\alpha|=1$. Let us notice that for every $j,\left|\beta_{j}\right| \leq 1 /\left|h\left(z^{j}\right)\right| \leq$ $1 / \delta$ and that

$$
\begin{aligned}
g(z) & =\vec{k} \cdot \sum_{j=1}^{N(r)}\left|\beta_{j}\right| \cdot e^{-m d^{2}\left(z, z^{\jmath}\right)} \cdot e^{i Q_{m, J}(z)} \\
& =\vec{k} \cdot \sum_{k=0}^{[\sqrt{2} / r]} \sum_{z^{\jmath} \in V_{k}(z)}\left|\beta_{j}\right| e^{-m d^{2}\left(z, z^{\jmath}\right)} e^{i Q_{m, J}(z)}
\end{aligned}
$$

for some real functions $Q_{m, j}$ and $\vec{k}=(0,0, \ldots, 0,1) \in \mathbf{C}^{m}$. 
If $V_{0}(z)=\varnothing$ or $z \in B\left(z^{j}, r\right)$ with $\beta_{J}=0$ then, because of (11) and the inequality $m r^{2}>2 n$, we have

$$
\begin{aligned}
|g(z)| & \leq \sum_{k=1}^{[\sqrt{2} / r]} \sum_{z^{J} \in V_{k}(z)} \frac{1}{\delta} e^{-m d^{2}\left(z, z^{J}\right)} \sum_{k=1}^{[\sqrt{2} / r]} \frac{1}{\delta}\left|V_{k}(z)\right| e^{-m k^{2} r^{2}} \\
& \leq \sum_{k=1}^{\infty} \frac{C_{3}}{\delta} k^{2 n-1} e^{-k^{2} m r^{2}} \leq \frac{C_{3}}{\delta} \sum_{k=1}^{\infty} e^{-k m r^{2}} \leq 2 \frac{C_{3}}{\delta} e^{-m r^{2}}=\varepsilon .
\end{aligned}
$$

This proves part (e) of Lemma 1.If $z \in B\left(z^{j}, r\right)$ with $\beta_{J} \neq 0$ then

$$
\begin{aligned}
& |v(z)+h(z) \cdot u(z)| \\
& \leq\left|v\left(z^{j}\right)+h\left(z^{j}\right) \cdot \beta_{j} \cdot e^{-m d^{2}\left(z, z^{\prime}\right)} \cdot e^{i Q_{m, J}(z)}\right| \\
& \quad+\left|\left[h(z)-h\left(z^{j}\right)\right] \cdot \beta_{j} \cdot e^{-m d^{2}\left(z, z^{j}\right)} \cdot e^{i Q_{m, J}(z)}\right|+\left|v(z)-v\left(z^{j}\right)\right| \\
& \quad+\left|h(z) \cdot \sum_{z^{\prime} \notin V_{0}(z)} \beta_{j} \cdot e^{-m d^{2}\left(z, z^{J}\right)} \cdot e^{i Q_{m, m}(z)}\right| \\
& =\mathrm{I}+\mathrm{II}+\mathrm{III}+\mathrm{IV} .
\end{aligned}
$$

Because of (9)

$$
\text { III } \leq \varepsilon \quad \text { and } \quad \text { II } \leq\left|h(z)-h\left(z^{j}\right)\right| \cdot\left|\beta_{j}\right|<\delta \cdot \varepsilon \cdot \frac{1}{\delta}=\varepsilon .
$$

By the same argument as in (12) we can prove that IV $\leq \varepsilon$. Moreover, we have $\mathrm{I} \leq\left|v\left(z^{j}\right)\right|+\left|h\left(z^{j}\right) \cdot \beta_{j}\right|=b$. This altogether gives us

$$
|v(z)+h(z) \cdot u(z)| \leq b+3 \varepsilon .
$$

Inequalities (12) and (14) prove part (a) of Lemma 1. Now we shall determine a certain subset $V$ of $W=\bigcup_{j=1}^{N(r)} B\left(z^{j}, r\right)$. To do this let us fix $j$, $1 \leq j \leq N(r)$, and let us take $\alpha=\left|v\left(z_{J}\right)\right|, s(z)=e^{-m d^{2}\left(z, z^{\prime}\right)}, Q(z)=$ $\arg \left(e^{-m\left(1-\left(\left\langle z, z^{j}\right\rangle\right)\right)}\right)=m \cdot \operatorname{Im}\left\langle z, z^{j}\right\rangle$.

Let us assume at first that $\alpha<1$. We define

$$
V_{j}=\left\{z \in B\left(z^{J}, r\right): s(z) \geq a \text { and } \cos Q(z) \geq a\right\}
$$

Using the same notation as in (13) we can write

$$
|v(z)+h(z) \cdot u(z)| \geq \mathrm{I}-\mathrm{II}-\mathrm{III}-\mathrm{IV} .
$$


As before, $\mathrm{II} \leq \varepsilon, \mathrm{III} \leq \varepsilon$ and $\mathrm{IV} \leq \varepsilon$. Assuming $z \in V_{\jmath}$, we have

$$
\begin{aligned}
\mathrm{I} & =\left|v\left(z^{j}\right)+h\left(z^{j}\right) \cdot \beta_{j} \cdot e^{-m \cdot d^{2}\left(z, z^{j}\right)} \cdot e^{l Q(z)}\right| \\
& \geq\left|\alpha+(1-\alpha) \cdot s(z) \cdot e^{l Q(z)}\right| \\
& =\sqrt{\alpha^{2}+2 \alpha(1-\alpha) \cdot s(z) \cdot \cos Q(z)+(1-\alpha)^{2}} \geq a
\end{aligned}
$$

because of our assumption about $s(z)$ and $\cos Q(z)$, the definition of $\beta_{J}$ and simple geometry.

Combining (15) and (16) we get

$$
|v(z)+h(z) \cdot u(z)|>a-3 \varepsilon \quad \text { for } z \in V_{j} .
$$

Let $\rho>0$ be defined by $m \rho^{2}=\log (1 / a)$. Then $\rho \leq r$ because $m r^{2}=$ $2 C_{3} / \delta \varepsilon$ and $2 C_{3} / \delta \geq 1 / a$. So $B\left(z^{J}, \rho\right) \subset B\left(z^{j}, r\right)$, and if $z \in B\left(z^{j}, \rho\right)$ then $s(z) \geq a$. The set $\left\{z \in B\left(z^{j}, \rho\right): \cos Q \geq a\right\}$ consists of certain strips in the ball $B\left(z^{\prime}, \rho\right)$. An easy geometric argument shows that these strips have a total area at least

$$
\frac{1}{2 \pi} \cdot \arccos a \cdot \sigma_{n}\left(B\left(z^{j}, \rho\right)\right)=\frac{1}{2 \pi} \cdot \arccos a \cdot A(\rho) .
$$

Moreover $V_{j} \subset B\left(z^{J}, r\right) \subset F_{\delta}$. Using inequality (4) and the fact that the above strips are included in $V_{J}$, we get

$$
\sigma_{n}\left(V_{j}\right) \geq \frac{1}{2 \pi} \cdot \arccos a \cdot A(\rho) \geq \frac{1}{2 \pi} \cdot A_{1} \cdot \arccos a \cdot \rho^{2 n-1}
$$

If $\alpha \geq 1$, we define $V_{j}=B\left(z^{J}, \rho\right)$. Because $\beta_{j}=0$, it follows from (12) that

$$
\begin{aligned}
|v(z)+h(z) \cdot u(z)| & \geq\left|v\left(z^{j}\right)\right|-\left|v(z)-v\left(z^{j}\right)\right|-|h(z) \cdot u(z)| \\
& \geq a-\varepsilon-|u(z)| \geq a-2 \varepsilon
\end{aligned}
$$

for $z \in V_{j}$.

Finally, we define $K=\bigcup_{j=1}^{N(r)} \bar{V}_{j}$. We observe that inequality (17) holds for $z \in K$. If $z \in P$, then $V_{0}(z)=\varnothing$ and inequality (12) gives us

$$
|v(z)+h(z) \cdot u(z)| \geq|v(z)|-|u(z)| \geq a-\varepsilon .
$$


This altogether proves part (c) of Lemma 1. It is easy to check that $K \cap P=\varnothing$. Inequalities (18), (6), (9) and the definitions of $\rho$ and $m r^{2}$ yield

$$
\begin{aligned}
\sigma_{n}( & K) \geq \sigma_{n}\left(\bigcup_{j=1}^{N(r)} V_{j}\right)=\sum_{j=1}^{N(r)} \sigma_{n}\left(V_{j}\right) \\
& \geq N(r) \cdot \frac{1}{2 \pi} \cdot A_{1} \cdot \arccos a \cdot \rho^{2 n-1} \\
& \geq \frac{C_{1}}{r^{2 n-1}} \cdot \sigma_{n}(S) \cdot \frac{1}{2 \pi} \cdot A_{1} \cdot \arccos a \cdot \rho^{2 n-1} \\
& \geq \frac{1}{4 \pi} \cdot C_{1} \cdot A_{1} \cdot \arccos a \cdot\left(m r^{2}\right)^{-(2 n-1) / 2} \cdot\left(m \rho^{2}\right)^{2 n-1} \cdot \sigma_{n}\left(F_{\delta}-P\right) \\
& =\varphi(a) \cdot \log \left(4 C_{3} /(\delta \varepsilon)\right)^{-(2 n-1) / 2} \cdot \sigma_{n}\left(F_{\delta}-P\right) .
\end{aligned}
$$

This proves part (d) of Lemma 1. Finally, if $|z| \leq R$ then $\operatorname{Re}\left(1-\left\langle z, z^{j}\right\rangle\right)$ $\leq 1-R$ for $j=1,2, \ldots, N(r)$. Because of the inequalities $m r^{2} \geq 1,(10)$ and (6), we have

$$
\begin{aligned}
|u(z)| & \leq N(r) \cdot e^{-m(1-R)} \leq C_{2} \cdot \frac{1}{r^{2 n-1}} \cdot e^{-m(1-R)} \\
& =C_{2} \cdot m^{(2 n-1) / 2} \cdot e^{-m(1-R)} \cdot\left(m r^{2}\right)^{-(2 n-1) / 2} \\
& \leq C_{2} \cdot m^{(2 n-1) / 2} \cdot e^{-m(1-R)} \leq \varepsilon .
\end{aligned}
$$

This proves part (d) of Lemma 1 and ends the proof.

LEMMA 2. Let $v$ be a continuous map $v: S^{n} \rightarrow \mathbf{C}^{m}$ such that $\|v\| \|_{\delta}<b$ $<1$ for some $\delta<C_{3}$. Let $\frac{1}{4}>\varepsilon>0, R<1$. Then there exists a holomorphic map u: $\mathbf{C}^{n} \rightarrow \mathbf{C}^{m}$ and a closed set $K \subset F_{\delta}$ such that:

(a) $\quad\|\| v+h \cdot u \|_{\delta}<b+\varepsilon$;

(b) $\quad\|u\|_{R} \leq \varepsilon$;

(c)' $|v(z)+h(z) \cdot u(z)|>b-\varepsilon$;

$(\mathrm{d})^{\prime} \quad \sigma_{n}(K) \geq \sigma_{n}\left(F_{\delta}\right)-\varepsilon$;

(e) $\quad|u(z)| \leq \varepsilon \quad$ for $z \in S^{n}-F_{\delta}$;

(f) $\quad u_{j} \equiv 0 \quad$ for $j=1,2, \ldots, m-1$, where $u=\left(u_{1}, u_{2}, \ldots, u_{m}\right)$.

Proof. Let $a=b-\frac{1}{2} \varepsilon$ and choose a sequence $\left\{\varepsilon_{j}\right\}$ satisfying the assumptions of Lemma 1 and such that $6 \sum_{j=1}^{\infty} \varepsilon_{j}<\varepsilon$. We can assume $\varepsilon_{j}=A \cdot \exp \left\{-(\tau \cdot j)^{2 / 2 n-1)}\right\}, A=2 C_{3} / \delta$ and $\tau$ is some large number. 
Apply Lemma 1 to the data $a, \varepsilon_{1}, R, v, P=\varnothing$ to produce a holomorphic map $u_{1}: \mathbf{C}^{n} \rightarrow \mathbf{C}^{m}$ and a closed set $K_{1} \subset F_{\delta}$ such that:

(a) $\quad\|\| v+h \cdot u_{1} \|_{\delta} \leq b+3 \varepsilon_{1}$;

(b) $\quad\left\|v_{1}\right\|_{R} \leq \varepsilon_{1}$;

(c) $1 \quad\left|v(z)+h(z) \cdot u_{1}(z)\right| \geq a-3 \varepsilon_{1} \quad$ for $z \in K_{1}$;

(d) $\quad \alpha_{1}=\sigma_{n}\left(K_{1}\right) \geq \varphi(a) \cdot\left[\log \left(A / \varepsilon_{1}\right)\right]^{-(2 n-1) / 2} \cdot \sigma_{n}\left(F_{\delta}\right)$;

(e) $\quad\left|u_{1}(z)\right| \leq \varepsilon_{1} \quad$ for $z \in S^{n}-F_{\delta}$;

(f) $u_{j}^{1} \equiv 0 \quad$ for $j=1,2, \ldots, m-1$, where $u_{1}=\left(u_{1}^{1}, u_{2}^{1}, \ldots, u_{m}^{1}\right)$.

Suppose that holomorphic maps $u_{1}, u_{2}, \ldots, u_{p-1}\left(u_{j}: \mathbf{C}^{n} \rightarrow \mathbf{C}^{m}\right.$ for $j=$ $1,2, \ldots, p-1)$ have been chosen together with closed sets $K_{1}, K_{2}, \ldots, K_{p-1}$ such that if $W_{i}=\bigcup_{j=1}^{i} K_{j}$ then $K_{i+1} \cap W_{i}=\varnothing$ and $\sigma_{n}\left(K_{i}\right)=\alpha_{i}, K_{i} \subset F_{\delta}$. A map $u_{p}: \mathbf{C}^{n} \rightarrow \mathbf{C}^{m}$ and a closed set $K_{p}$ is then obtained by applying Lemma 1 to the data $a-3 \sum_{i=1}^{p-1} \varepsilon_{i}, \varepsilon_{p}, R, v+h(z) \cdot\left(u_{1}+u_{2}+\cdots+\right.$ $\left.u_{p-1}\right), W_{p-1}$. This produces a sequence $\left\{v_{k}\right\}$ of holomorphic maps $\left(v_{k}\right.$ : $\mathbf{C}^{n} \rightarrow \mathbf{C}^{m}$ for $\left.k=1,2, \ldots\right)$ and a sequence $\left\{K_{k}\right\}$ of disjoint closed sets such that $K_{k} \subset F_{\delta}, \sigma_{n}\left(K_{k}\right)=\alpha_{k}$ and:

(a) $p$

$$
\left\|v+h \cdot \sum_{k=1}^{p} u_{k}\right\|_{\delta} \leq b+3 \cdot \sum_{k=1}^{p} \varepsilon_{k}<b+\varepsilon ;
$$

(b) $p$

$$
\left\|\sum_{k=1}^{p} u_{k}\right\|_{R} \leq \sum_{k=1}^{p}\left\|u_{k}\right\|_{R} \leq \sum_{k=1}^{p} \varepsilon_{k}<\varepsilon
$$

(c) $p$

$$
\begin{aligned}
\left|v(z)+h(z) \cdot \sum_{k=1}^{p} u_{k}(z)\right| & \geq a-3 \cdot \sum_{k=1}^{p} \varepsilon_{k} \\
& \geq a-\frac{1}{2} \varepsilon=b-\varepsilon \quad \text { for } z \in W_{p} ;
\end{aligned}
$$

$(\mathrm{d})_{p} \quad \alpha_{p}=\sigma_{n}\left(K_{p}\right)$

$$
\begin{aligned}
& \geq \varphi\left(a-3 \cdot \sum_{k=1}^{p-1} \varepsilon_{i}\right) \cdot\left[\log \frac{A}{\varepsilon_{p}}\right]^{-(2 n-1) / 2} \cdot\left(\sigma_{n}\left(F_{\delta}\right)-\sum_{k=1}^{p-1} \alpha_{k}\right) \\
& \geq \varphi(a) \cdot\left[\log \frac{A}{\varepsilon_{p}}\right]^{-(2 n-1) / 2} \cdot\left(\sigma_{n}\left(F_{\delta}\right)-\sum_{k=1}^{p-1} \alpha_{k}\right) ;
\end{aligned}
$$

(e) $p \quad\left|\sum_{k=1}^{p} u_{k}(z)\right| \leq \sum_{k=1}^{p}\left|u_{k}(z)\right| \leq \sum_{k=1}^{p} \varepsilon_{k}<\varepsilon \quad$ for $z \in S^{n}-F_{\delta}$;

$$
u_{j}^{k} \equiv 0 \quad \text { for } k=1,2, \ldots, p \text { and } j=1,2, \ldots, m-1,
$$

$$
\text { where } u_{k}=\left(u_{1}^{k}, u_{2}^{k}, \ldots, u_{m}^{k}\right) \text {. }
$$


If $\sum_{k=1}^{\infty} \alpha_{k}<\sigma_{n}\left(F_{\delta}\right)$, (d) shows that there is a constant $C_{4}$ such that for every positive integer $k$,

$$
\alpha_{p} \geq C_{4} \cdot\left[\log \frac{A}{\varepsilon_{p}}\right]^{-(2 n-1) / 2}=\left[C_{4} \cdot(\tau p)^{2 /(2 n-1)}\right]^{-(2 n-1) / 2}=\frac{C_{4}}{\tau p} .
$$

This is impossible, because then $\sum_{p=1}^{\infty} \alpha_{p}=\infty$ and $\alpha_{p}$ are the measures of the disjoint sets. Hence, we may assume that $\sum_{k=1}^{\infty} \alpha_{k}=\sigma_{n}\left(F_{\delta}\right)$. It follows that for $p$ sufficiently large and $P=W_{p}$ we have $\sigma_{n}(P)=\sum_{k=1}^{p} \alpha_{k}>1-\varepsilon$, which is part (d)' of Lemma 2. Letting $h=\sum_{k=1}^{p} u_{k}$, parts (a)', (b)', (c)', $(\mathrm{e})^{\prime},(\mathrm{f})^{\prime}$ are just $(\mathrm{a})_{p},(\mathrm{~b})_{p},(\mathrm{c})_{p},(\mathrm{e})_{p},(\mathrm{f})_{p}$. So we have proved the assertion of Lemma 2.

Assume now that $g$ and $h$ satisfy the assumptions of the Theorem. Then $\||g|\|_{\delta} \leq 1-\delta$. To prove the Theorem, take a sequence $\delta_{1}, \delta_{2}, \ldots$ of positive numbers such that $\delta_{1}<C_{3}$ and $\delta_{i+1}<\delta_{l} / 2$ and let $a_{1}=b_{1}=1-$ $\frac{1}{2} \delta_{1}, \varepsilon_{1}=\min \left(\frac{1}{16}, \frac{1}{4} \delta_{1}\right), R_{1}=\frac{1}{2}$. Apply Lemma 2 to the data $g_{1}=g, b_{1}, \delta_{1}$, $R_{1}$ to get a map $u_{1}$ and a set $K_{1} \subset F_{\delta_{1}}$ such that, for $p=1$ and $g_{1}=g$ :

(i) $\quad \quad\left\|g_{p}+h \cdot u_{p}\right\| \|_{\delta_{p}}<b_{p}+\varepsilon_{p}<1$;

(ii) ${ }_{p} \quad\left\|u_{p}\right\|_{R_{p}} \leq \varepsilon_{p}$;

(iii) $_{p} \quad\left|g_{p}(z)+h(z) \cdot u_{p}(z)\right|>b_{p}-\varepsilon_{p} \quad$ for $z \in K_{p}$;

(iv) $p \quad \sigma_{n}\left(K_{p}\right) \geq \sigma_{n}\left(F_{\delta_{p}}\right)-\varepsilon_{p}$;

$(\mathrm{v})_{p} \quad 1-\left|g_{p}(z)+h(z) \cdot u_{p}(z)\right|$

$$
\geq\left(1-\sum_{i=1}^{p} \varepsilon_{i}\right)|h(z)| \quad \text { for } z \in S^{n}-F_{\delta_{p}} ;
$$

(vi) $u_{p} \quad u_{j}^{p} \equiv 0 \quad$ for $j=1,2, \ldots, m-1$ where $u_{p}=\left(u_{1}^{p}, u_{2}^{p}, \ldots, u_{m}^{p}\right)$.

Inequality (v) follows from (e) of Lemma 2, because for $z \in S^{n}-F_{\delta_{1}}$, we have $\left|u_{1}(z)\right|<\varepsilon_{1}$, so

$$
\begin{aligned}
1-\left|v(z)+h(z) \cdot u_{1}(z)\right| & \geq 1-|v(z)|-\left|u_{1}(z) \cdot h(z)\right| \\
& \geq|h(z)|-\varepsilon_{1} \cdot|h(z)|=\left(1-\varepsilon_{1}\right) \cdot|h(z)| .
\end{aligned}
$$

Since $g_{1}+h \cdot u_{1}$ is a continuous map on $\bar{B}^{n}$, there exists an $R_{2}$ such that $\frac{1}{2}+\frac{1}{2} R_{1}<R_{2}<1$ and, for $p=1$,

$(\text { vii })_{p} \quad\left|g_{p}\left(R_{p+1} \cdot z\right)+h\left(R_{p+1} \cdot z\right) \cdot u_{p}\left(R_{p+1} \cdot z\right)\right|>b_{p}-2 \varepsilon_{p}$ 
Suppose we have inductively found holomorphic maps $u_{1}, u_{2}, \ldots, u_{p}$, closed sets $K_{1}, K_{2}, \ldots, K_{p}$, real numbers $R_{1}, R_{2}, \ldots, R_{p+1}, b_{1}, b_{2}, \ldots, b_{p}$, $\varepsilon_{1}, \varepsilon_{2}, \ldots, \varepsilon_{p}$ such that $\frac{1}{2}+\frac{1}{2} R_{l}<R_{i+1}, \varepsilon_{l}>0$ for $i=1,2, \ldots, p$ and $\sum_{i=1}^{p} \varepsilon_{i}<1 / 8$. Let us assume $g_{J+1}=g+h \cdot \sum_{i=1}^{J} u_{i}$ and conditions (i) $j-$ (vii) $)_{j}$ are satisfied for $j=1,2, \ldots, p$. We also assume that $1-1 / j \leq$ $b_{J}<b_{J}+\varepsilon_{J}<1$. If $z \in\left(F_{\delta_{p+1}}-F_{\delta_{p}}\right)$ then according to $(\mathrm{v})_{p}$, we have

$$
1-\left|g_{p+1}(z)\right| \geq\left(1-\sum_{i=1}^{p} \varepsilon_{l}\right) \cdot|h(z)| \geq \frac{1}{2} \cdot \delta_{p+1}
$$

since $|h(z)| \geq \delta_{p+1}$. This, together with (i) $)_{p}$, shows that $\left\|\left|g_{p+1}\right|\right\|_{\delta_{p+1}}<1$. Take any $b_{p+1}>1-1 /(p+1)$ and $\varepsilon_{p+1}$ satisfying the inequalities $1>$ $b_{p+1}+\varepsilon_{p+1}>b_{p+1}>\left\||| g_{p+1} \mid\right\|_{\delta_{p+1}}$ and $\sum_{i=1}^{p+1} \varepsilon_{i}<1 / 8$. Since the map $g_{p+1}$ is continuous on $\bar{B}^{n}$, we can find a number $R_{p+2}$ such that $\frac{1}{2}+\frac{1}{2} R_{p+1}<$ $R_{p+2}<1$ and such that condition (vii) $)_{p+1}$ is satisfied. Now we can apply Lemma 2 to the data $g_{p+1}, b_{p+1}, \varepsilon_{p+1}, R_{p+1}$. We get some map $u_{p+1}$ and a set $K_{p+1}$. It follows from Lemma 2 that conditions (i) ${ }_{p+1}-(\text { iv) })_{p+1}$ and (vi) $_{p+1}$ are satisfied. For $z \in S^{n}-F_{\delta_{p+1}}$, by the virtue of $(\mathrm{e})^{\prime}$ and $(\mathrm{v})_{p}$, we have

$$
\begin{aligned}
1-\mid g_{p+1}( & (z)+h(z) \cdot u_{p+1}(z) \mid \\
\geq & 1-\left|g_{p}(z)+h(z) \cdot u_{p}(z)\right|-\left|h(z) \cdot u_{p+1}(z)\right| \\
\geq & \left(1-\sum_{i=1}^{p} \varepsilon_{i}\right) \cdot|h(z)|-|h(z)| \cdot \varepsilon_{p+1} \\
= & \left(1-\sum_{i=1}^{p+1} \varepsilon_{i}\right) \cdot|h(z)| .
\end{aligned}
$$

So we have also proved that condition $(\mathrm{v})_{p+1}$ is satisfied. Conditions (ii) $)_{p}$ $(p=1,2,3 \ldots)$ and the definition of $g_{p}$ say that the sequence $\left\{g_{p}\right\}$ is convergent uniformly on every ball $R_{p} \cdot B^{n}$, and since $\lim _{p \rightarrow 1} R_{p}=1$, this sequence is pointwise convergent to some holomorphic map $f$ on the ball $B^{n}$. From conditions (i) $)_{p}$ and $(\mathrm{v})_{p}$ it follows that each map $g_{p}$ is bounded by 1 on $B^{n}$. So, also $\|f\|_{\infty} \leq 1$. For $\delta>0$ let $L_{p}=F_{\delta} \cap \cap_{J>p} K_{J}$. Then, for $q$ large enough, $F_{\delta} \subset F_{\delta_{p}}$ for $p>q$. We have

$$
\begin{aligned}
\sigma_{n}\left(F_{\delta}\right)-\sigma_{n}\left(L_{q}\right) & =\sigma_{n}\left(\bigcup_{j>q}\left(F_{\delta}-\left(F_{\delta} \cap K_{q}\right)\right)\right) \\
& \leq \sum_{j>q} \sigma_{n}\left(F_{\delta}-\left(F_{\delta} \cap K_{j}\right)\right) \leq \sum_{j>q} \sigma_{n}\left(F_{\delta_{j}}-K_{j}\right)<\sum_{j>q} \varepsilon_{j} .
\end{aligned}
$$


Hence $\lim _{q \rightarrow \infty} \sigma_{n}\left(L_{q}\right)=\sigma_{n}\left(F_{\delta}\right)$. It is obvious from (iii) $p$ and the equality $\lim _{p \rightarrow \infty} b_{p}=1$ that $\lim _{R \rightarrow 1} f(R z)=1$ for $z \in L_{q}$, provided this limit exists. Since $\delta$ was arbitrary, this proves that the map $f$ is inner, since $\sigma_{n}\left(\cap_{p}\left(S^{n}-F_{\delta_{p}}\right)\right)=0$. Now it is easy to check that $f$ satisfies the Theorem.

COROLlaRY 3. Let $m<n$ and let $g \in A_{m}\left(B^{m}\right),\|g\|_{\infty} \leq 1$. There exists an inner map $f: B^{n} \rightarrow B^{m}$ such that

$$
f\left(z_{1}, z_{2}, \ldots, z_{m}, 0,0, \ldots, 0\right)=g\left(z_{1}, z_{2}, \ldots, z_{m}\right) .
$$

Proof. Let $\Phi: B^{m} \rightarrow B^{m}$ be an automorphism of $B^{m}$ such that $\Phi(g(0, \ldots, 0))=(0, \ldots, 0)$. Take $\tilde{g}: B^{m} \rightarrow B^{m}, \tilde{g}(z)=\Phi\left(g\left(z_{1}, z_{2}, \ldots, z_{m}\right)\right)$, $h(z)=\frac{1}{2} \cdot z_{n}^{2}$. By virtue of Schwartz's lemma,

$$
\tilde{g}(z) \leq\left(\left|z_{1}\right|^{2}+\left|z_{2}\right|^{2}+\cdots+\left|z_{m}\right|^{2}\right)^{1 / 2} \text {. }
$$

So we have

$$
|\tilde{g}(z)|+|h(z)| \leq\left(1-\left|z_{n}\right|^{2}\right)^{1 / 2}+\frac{1}{2} \cdot\left|z_{n}\right|^{2} \leq 1
$$

We can apply the Theorem for $g$ and $h$ to get an inner map $\tilde{f}$. The inner map $f=\Phi^{-1}(\tilde{f})$ will satisfy Corollary 3 .

COROLlaRY 4. There exists an inner function $f: B^{n} \rightarrow D$ such that

$$
\frac{\partial f}{\partial z_{1}}(0,0, \ldots, 0)=1 \text {. }
$$

Proof. Take $m=1$ in Corollary 3 and a function $g: B^{1} \rightarrow D, g(z)=z$.

REMARK. The assumption $g \in A_{m}\left(B^{m}\right)$ in Corollary 3 is not necessary: we can take any holomorphic map $g: B^{m} \rightarrow B^{m}$. Then the map $\tilde{g}$, defined as before, can be prolonged to a continuous map on $\bar{B}^{n}-A$, where $A \subset S^{n}$ and $\sigma_{n}(A)=0$. One can check that the Theorem is still valid for such maps. 


\section{REFERENCES}

[1] A. B. Aleksandrov, The existence of inner functions on the unit ball, Mat. Sb. 1982, v. 118 (160) N2(6), 147-163 (in Russian).

[2] E. Löw, $A$ construction of inner functions on the unit ball of $\mathbf{C}^{p}$, Invent. Math., 67 (1982), 223-229.

[3] W. Rudin, Inner functions in the unit ball, preprint.

[4] _ Function Theory in the Unit Ball of $\mathbf{C}^{n}$, Springer, New York, 1980.

Received February 11, 1983.

UNIVERSITY OF WISCONSIN

MADISON, WI 53706 



\title{
PACIFIC JOURNAL OF MATHEMATICS \\ EDITORS
}

\author{
DoNALD BABBITT (Managing Editor) \\ University of California \\ Los Angeles, CA 90024 \\ J. DugunduI \\ University of Southern California \\ Los Angeles, CA 90089-1113 \\ R. FINN \\ Stanford University \\ Stanford, CA 94305 \\ HERMANN FlasChKa \\ University of Arizona \\ Tucson, AZ 85721
}

C. C. Moore

University of California

Berkeley, CA 94720

ARTHUR OGUS

University of California

Berkeley, CA 94720

Hugo Rossi

University of Utah

Salt Lake City, UT 84112

H. SAMELSON

Stanford University

Stanford, CA 94305

ASSOCIATE EDITORS
R. ARENS
E. F. BECKENBACH
B. H. NeUMANN
F. WOLF
K. YoSHIDA (1906-1982)

\section{SUPPORTING INSTITUTIONS}

UNIVERSITY OF ARIZONA
UNIVERSITY OF BRITISH COLUMBIA
CALIFORNIA INSTITUTE OF TECHNOLOGY
UNIVERSITY OF CALIFORNIA
MONTANA STATE UNIVERSITY
UNIVERSITY OF NEVADA, RENO
NEW MEXICO STATE UNIVERSITY
OREGON STATE UNIVERSITY
UNIVERSITY OF ARIZONA
CALIFORNIA INSTITUTE OF TECHNOLOGY
UNIVERSITY OF CALIFORNIA
UNIVERSITY OF NEVADA, RENO
OREGON STATE UNIVERSITY
UNIVERSITY OF OREGON
UNIVERSITY OF SOUTHERN CALIFORNIA
STANFORD UNIVERSITY
UNIVERSITY OF HAWAII
UNIVERSITY OF TOKYO
UNIVERSITY OF UTAH
WASHINGTON STATE UNIVERSITY
UNIVERSITY OF WASHINGTON 


\section{Pacific Journal of Mathematics}

Vol. 115, No. $1 \quad$ September, 1984

Carlos Andradas Heranz and José Manuel Gamboa Mutuberría, A note on projections of real algebraic varieties $\ldots \ldots \ldots \ldots \ldots \ldots \ldots \ldots \ldots \ldots$

Jürgen Appell and Maria Patrizia Pera, Noncompactness principles in nonlinear operator approximation theory $\ldots \ldots \ldots \ldots \ldots \ldots \ldots \ldots \ldots \ldots$

Timothy John Carlson, Extending Lebesgue measure by infinitely many

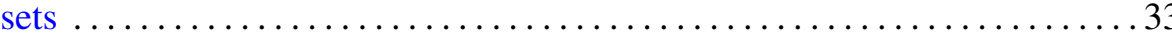

Donald S. Coram and Paul Frazier Duvall, Jr., Non-cell-like

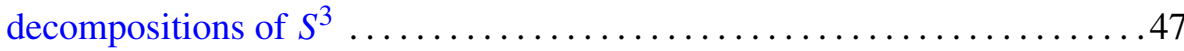

Edward Norman Dancer, Order intervals of selfadjoint linear operators and

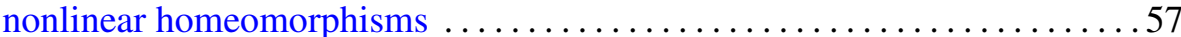

Ralph Jay De Laubenfels, Well-behaved derivations on $C[0,1] \ldots \ldots \ldots 73$

D. Feyel and A. de La Pradelle, Sur certaines extensions du théorème

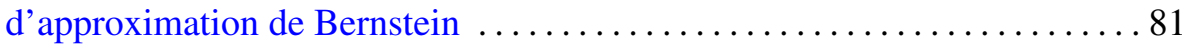

Colin C. Graham and Bertram Manuel Schreiber, Bimeasure algebras on

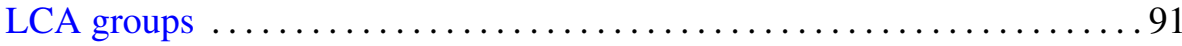

Richard Howard Hudson, Class numbers of imaginary cyclic quartic fields

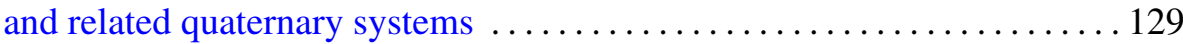

Carl Groos Jockusch, Jr. and Iraj Kalantari, Recursively enumerable sets and van der Waerden's theorem on arithmetic progressions . . . . . . . . 143

J. F. McClendon, On noncontractible valued multifunctions ........... 155 Akihiko Miyachi, Weak factorization of distributions in $H^{p}$ spaces $\ldots \ldots \ldots 165$ Ezzat S. Noussair and Charles Andrew Swanson, Global positive solutions of semilinear elliptic problems

Jon Christopher Snader, Strongly analytic subspaces and strongly

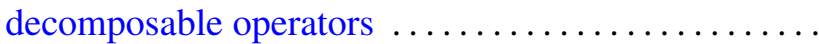

Boguslaw Tomaszewski, A construction of inner maps preserving the Haar measure on spheres

Akihito Uchiyama, The Fefferman-Stein decomposition of smooth functions and its application to $H^{p}\left(\mathbf{R}^{n}\right) \ldots \ldots \ldots$ 